\title{
Reflection of Magnetoelastic Waves on the Boundary of Two Ferromagnetic Media with Different Strength of Coupling in the Interface
}

\author{
Yu.I. Gorobets, S.A. Reshetnyak and T.A. Khomenko* \\ National Technical University of Ukraine "Kiev Polytechnic Institute", 37 Peremohy av., 03056 Kiev, Ukraine \\ The theoretical research of reflection of bulk magnetoelastic waves in the ferromagnet with isotropic elastic \\ properties has been investigated. It shows calculation of intensities of reflection and transmission of magnetoelastic \\ waves at falling on the boundary of two semi-infinite uniform ferromagnets with different values of parameters \\ of exchange interaction, uniaxial magnetic anisotropy and saturation magnetization; taking into consideration \\ the coupling in the interface, which qualitatively changes the nature of frequency dependences of reflected \\ magnetoelastic waves intensity and dependences on the external uniform permanent magnetic field.
}

PACS numbers: $72.55 .+\mathrm{s}$

\section{Introduction}

Ferromagnetic materials are unique objects for both experimental and theoretical research and have a range of important features including interaction of spin and elastic waves. This interaction in magnetically ordered medium results in magnetoelastic waves. The problem of magnetoelastic interaction is interesting both from the side of studying of the magnetism nature and from the side of applying of this effect in engineering.

The work [1] presents a brief review of investigations of the behaviour of magnetoelastic waves in ferromagnetic plates and films. Transmission of surface magnetoelastic waves in semi-infinite ferromagnets is analyzed in the works $[2,3]$. Reflection of bulk magnetoelastic waves from the surface of the semi-infinite ferromagnet with the magnetization parallel to the surface is theoretically explored in the work [4].

We should note that properties of magnetoelastic waves of a certain frequency propagating in magnetic artificial two-layer ferromagnetic structure qualitatively differ from those observed at waves propagating in uniform ferromagnets, so the work [5] considers the behaviour of magnetoelastic waves in the two- and three-layer structures with the type of the magnetic-non-magnetic substrate. The work [6] describes propagation of magnetoelastic waves in the system consisting of the non-magnetic substrate covered with the metallic ferromagnetic film and solve a boundary-value problem for the indicated structure.

The works [7-10] consider the problems of reflection and transmission of magnetoelastic waves on the boundary of two magnetics and on the boundary of the mag-

* corresponding author; e-mail: homenko_t@ukr.net netic and non-magnetic matter. The work [11] presents results of experimental data concerning reflection features of magnetoelastic waves in the amorphous wire FeSiB.

In this work we explore theoretically the behaviour of magnetoelastic waves falling on the boundary of two uniform uniaxial ferromagnets with different values of parameters of exchange interaction, uniaxial magnetic anisotropy and saturation magnetization. We consider magnetoelastic waves within the framework of geometrical optics as rays (it is possible at the certain characteristic size of the medium inhomogeneities).

\section{Basic equations}

Let us consider two semi-infinite ferromagnets, the magnetizations of which are correspondingly $M_{01}$ and $M_{02}$, the parameters of exchange interaction are $\alpha_{1}$ and $\alpha_{2}$, and the parameters of uniaxial magnetic anisotropy are $\beta_{1}, \beta_{2}$. Let us choose the coordinate system so that the contact plane of ferromagnets is parallel to the plane $x y$ and the external uniform permanent magnetic field $\boldsymbol{H}_{0}$ is directed along the easy axis of ferromagnet magnetization and the axis $z$ of the selected coordinate system.

In accordance with [12], the linearized equations describing the transmission of magnetoelastic waves of small amplitudes in the exchange mode are the following:

$$
\begin{aligned}
& \frac{\partial \boldsymbol{\mu}}{\partial t}=g \mu_{0}\left(\boldsymbol{e}_{z} \times \boldsymbol{H}_{\mathrm{e}}\right), \quad \rho_{0} \frac{\partial^{2} \boldsymbol{u}}{\partial t^{2}}=\boldsymbol{F}, \\
& \frac{\partial \rho}{\partial t}+\rho_{0} \operatorname{div} \boldsymbol{v}=0,
\end{aligned}
$$

where $\boldsymbol{\mu}$ is a small deviation of the magnetic moment from the equilibrium value $\mu_{0}, g$ is a gyromagnetic ratio, 
$\boldsymbol{H}_{\mathrm{e}}$ is the effective magnetic field, $\rho$ is a small deviation from the equilibrium value of density $\rho_{0}, \boldsymbol{u}$ is a vector of elastic displacement, $\boldsymbol{F}$ is density of elastic force, $\boldsymbol{v}$ is a velocity of a ferromagnet element, $\boldsymbol{e}_{z}$ is a unit vector directed along the easy axis of ferromagnet magnetization.

The components of the effective magnetic field are determined according to the following formulae:

$$
\begin{aligned}
& H_{\mathrm{e} j}=\rho_{0}\left(\alpha \Delta-\beta-\frac{H_{0}}{M_{0}}\right) \mu_{j} \\
& -\rho_{0} \mu_{0}\left[(f+\beta) \frac{\partial u_{z}}{\partial x_{j}}+f \frac{\partial u_{j}}{\partial z}\right], \\
& j=x, y .
\end{aligned}
$$

$f$ is a constant that is usually: $f \approx 3-10$.

Expressions for components of elastic force density are the following [12]:

$$
\begin{gathered}
F_{j}=\left[\rho_{0}\left(s_{\ell}^{2}-2 s_{\mathrm{t}}^{2}\right) \delta_{j k} \delta_{m n}+\rho_{0} s_{\mathrm{t}}^{2}\left(\delta_{j m} \delta_{k n}-\delta_{j n} \delta_{k m}\right)\right. \\
\left.+\left(\mu_{0} \rho_{0}\right)^{2}(f+\beta) \delta_{j z} \delta_{j m} \delta_{k n}-\left(\mu_{0} \rho_{0}\right)^{2} f \delta_{j m} \delta_{k n}\right] \\
\quad \times \frac{\partial^{2} u_{m}}{\partial x_{k} \partial x_{n}}+\mu_{0} \rho_{0}^{2}\left[(f+\beta) \delta_{j z} \frac{\partial \mu_{m}}{\partial x_{m}}+f \frac{\partial \mu_{j}}{\partial z}\right]
\end{gathered}
$$

where $s_{\ell}$ and $s_{\mathrm{t}}$ are the velocities of longitudinal and transverse sound.

According to the JWKB method described in [13], a magnetoelastic wave propagating along the axis $z$, which is perpendicular to the media boundary, can be described by the following expressions:

$$
\begin{aligned}
& \boldsymbol{\mu}(\boldsymbol{r}, t)=\boldsymbol{\mu} \exp \left(\mathrm{i} k_{1} \psi(\boldsymbol{r})-\omega t\right), \\
& \boldsymbol{u}(\boldsymbol{r}, t)=\boldsymbol{u} \exp \left(\mathrm{i} k_{1} \psi(\boldsymbol{r})-\omega t\right), \\
& \rho(\boldsymbol{r}, t)=\rho \exp \left(\mathrm{i} k_{1} \psi(\boldsymbol{r})-\omega t\right),
\end{aligned}
$$

where $\omega$ is a velocity of a magnetoelastic wave, $k_{1}$ is a module of a wave vector in the medium, where an incident wave is transmitted, $\psi(\boldsymbol{r})$ is a wave function (eikonal).

Consequently, at satisfying the geometrical optics condition $\lambda \ll d$ (here $\lambda$ is a wavelength, $d$ is a characteristic size of heterogeneities) we obtain for circular components $\mu^{ \pm}=\mu_{x} \pm \mathrm{i} \mu_{y}, u^{ \pm}=u_{x} \pm \mathrm{i} u_{y}$ from the Eq. (1) taking into consideration (2)-(4):

$$
\begin{aligned}
& a_{j}\left(k_{1}^{ \pm} \nabla \psi^{ \pm}\right)^{4}+b_{j}^{ \pm}\left(k_{1}^{ \pm} \nabla \psi^{ \pm}\right)^{2}+c_{j}^{ \pm}=0, \\
& \quad j=1,2,
\end{aligned}
$$

where

$$
\begin{aligned}
a_{j} & =\alpha_{j} g M_{0} s_{\mathrm{t}}^{2}, \\
b_{j}^{ \pm} & =g M_{0} s_{\mathrm{t}}^{2}\left(\beta_{j}+H_{0} / M_{0}\right) \\
& -\alpha_{j} g M_{0} \omega^{2} \mp s_{\mathrm{t}}^{2} \omega-g f^{2} M_{0}^{3} / \rho_{0}, \\
c_{j}^{ \pm} & = \pm \omega^{3}-\omega^{2} g M_{0}\left(\beta_{j}+H_{0} / M_{0}\right) .
\end{aligned}
$$

The square of a wave vector has two values in each medium both for left-polarized wave $\left(\boldsymbol{\mu}^{+}, \boldsymbol{u}^{+}\right)$and for right-polarized wave $\left(\boldsymbol{\mu}^{-}, \boldsymbol{u}^{-}\right)$:

$$
\begin{aligned}
& \left(k_{j}^{ \pm}\right)_{A}^{2}=\left(-b_{j}^{ \pm}+\sqrt{\left(b_{j}^{ \pm}\right)^{2}-4 a_{j} c_{j}^{ \pm}}\right) / 2 a_{j}, \\
& \left(k_{j}^{ \pm}\right)_{B}^{2}=\left(-b_{j}^{ \pm}-\sqrt{\left(b_{j}^{ \pm}\right)^{2}-4 a_{j} c_{j}^{ \pm}}\right) / 2 a_{j} .
\end{aligned}
$$

Taking into consideration (7), the Eq. (5) can be written as

$$
\left[\left(\nabla \psi^{ \pm}\right)^{2}-\left(n_{j}^{ \pm}\right)_{A}^{2}\right]\left[\left(\nabla \psi^{ \pm}\right)^{2}-\left(n_{j}^{ \pm}\right)_{B}^{2}\right]=0,
$$

where

$$
\left(n_{j}^{ \pm}\right)_{A, B}^{2}=\left(k_{j}^{ \pm}\right)_{A, B}^{2} /\left(k_{1}^{ \pm}\right)_{A, B}^{2} .
$$

The square of the value $n_{j}^{ \pm}$equals to the relation of squares of wave vectors, therefore $n_{j}^{ \pm}$is a refraction index for $j$ medium in relation to the first medium. It is obvious that $n_{1}^{ \pm}=1$.

\section{Intensities of reflection and transmission}

To determine the reflection amplitude or reflection coefficient of a magnetoelastic wave and the amplitude of transmission through the boundary of two media, we will write: an incident wave as $\mu^{ \pm}(\boldsymbol{r}, t)=$ $\exp \left(\mathrm{i} k_{1}^{ \pm} \psi^{ \pm}(\boldsymbol{r})-\omega t\right)$, a reflected wave as $\mu_{R}^{ \pm}(\boldsymbol{r}, t)=$ $R^{ \pm} \exp \left(-\mathrm{i} k_{1}^{ \pm} \psi^{ \pm}(\boldsymbol{r})-\omega t\right)$, and a transmitted wave as $\mu_{D}^{ \pm}(\boldsymbol{r}, t)=D^{ \pm} \exp \left(\mathrm{i} k_{1}^{ \pm} \psi^{ \pm}(\boldsymbol{r})-\omega t\right)$.

For the material consisting of two uniform parts that contact along the plane $x y$, energy density can be written as

$$
w=\sum_{j=1}^{2} \theta\left[(-1)^{j} z\right] w_{j}+A \delta(z) \boldsymbol{M}_{1} \boldsymbol{M}_{2},
$$

where

$$
\begin{aligned}
& w_{j}=\frac{1}{8 \pi} H^{2}+\frac{1}{2} \rho_{0} v_{j}^{2}+\frac{1}{2} \lambda_{j, l m ; l^{\prime} m^{\prime}} \frac{\partial u_{j l}}{\partial x_{m}} \frac{\partial u_{j l^{\prime}}}{\partial x_{m^{\prime}}} \\
& +\frac{1}{2} \rho_{0}^{2} \alpha_{j}\left(\frac{\partial \boldsymbol{\mu}_{j}}{\partial x_{k}}\right)^{2}+\frac{1}{2} f \rho_{0}^{2} \mu_{0 j}^{2}\left(n_{l} n_{l^{\prime}} \delta_{m m^{\prime}}-n_{m} n_{m^{\prime}} \delta_{l l^{\prime}}\right) \\
& \quad \times \frac{\partial u_{j l}}{\partial x_{m}} \frac{\partial u_{j l^{\prime}}}{\partial x_{m^{\prime}}}+\frac{1}{2} \beta_{j} \rho_{0}^{2} \mu_{0 j}^{2}\left(\frac{\partial u_{j z}}{\partial x_{m}}\right)^{2} \\
& +\rho_{0}^{2} \mu_{0 j}\left[(f+\beta) \boldsymbol{\mu}_{j} \frac{\partial u_{j z}}{\partial x_{m}}+f \boldsymbol{\mu}_{j} \frac{\partial u_{j m}}{\partial z}\right]+\frac{1}{2} \rho_{0}^{2} \beta_{j} \mu_{j}^{2} \\
& \quad j=1,2
\end{aligned}
$$

$\theta(z)$ is a Heaviside function, $A$ is a parameter characterizing the coupling in the interface between uniform parts and measured in units of length. The case of $A=0$ corresponds to the absence of the coupling in the interface, and $A \rightarrow \infty$ corresponds to an ideal (in exchange sense) boundary. Change of the parameter value can be interpreted as a change of effective distance between adjacent layers, due to which the layer-to-layer exchange either increases $(A \rightarrow \infty)$ or decreases $(A \rightarrow 0)$. The estimations show that the parameter $A$ has the order of 


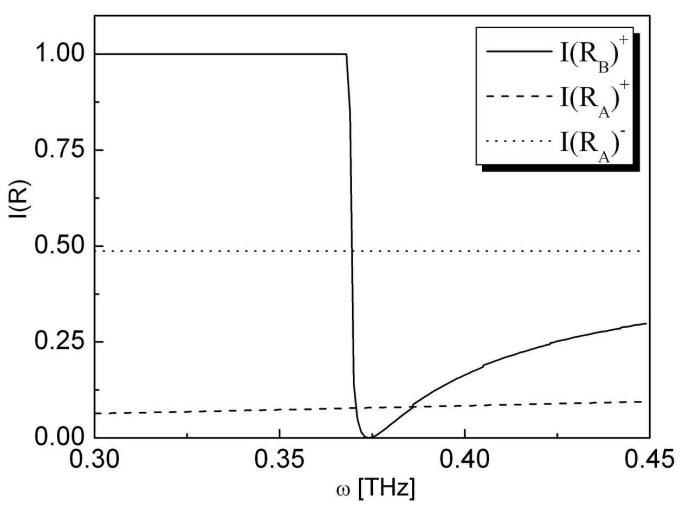

Fig. 1. Dependence of reflection intensity, for each component of reflected wave, on the magnetoelastic wave frequency at: $f=5, \alpha_{2} / \alpha_{1}=5.5, \beta_{2} / \beta_{1}=3.73$, $\gamma=M_{02} / M_{01}=0.95(T=300 \mathrm{~K}), H_{0}=10^{3}$ Oe, $s_{\mathrm{t}}=3 \times 10^{5} \mathrm{~cm} / \mathrm{s}, \rho_{0}=10 \mathrm{~g} / \mathrm{cm}^{3}, A=10^{-4} \mathrm{~cm}$.

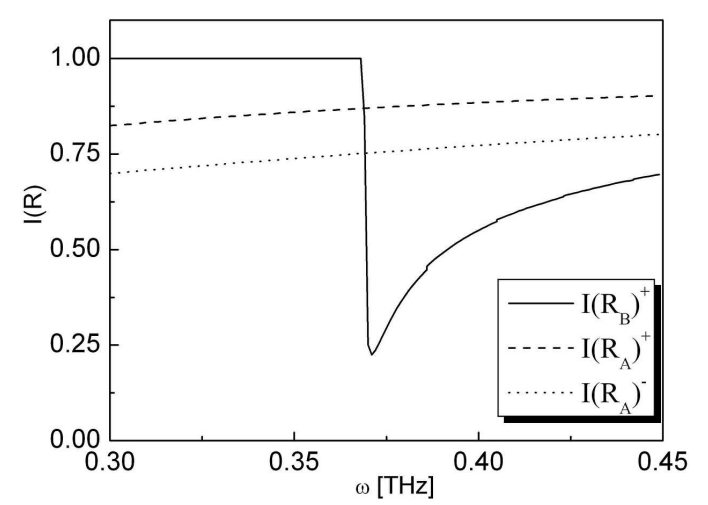

Fig. 2. Dependence of reflection intensity, for each component of reflected wave, on the magnetoelastic wave frequency at $A=10^{-6} \mathrm{~cm}$. All other parameters are the same as in Fig. 1.

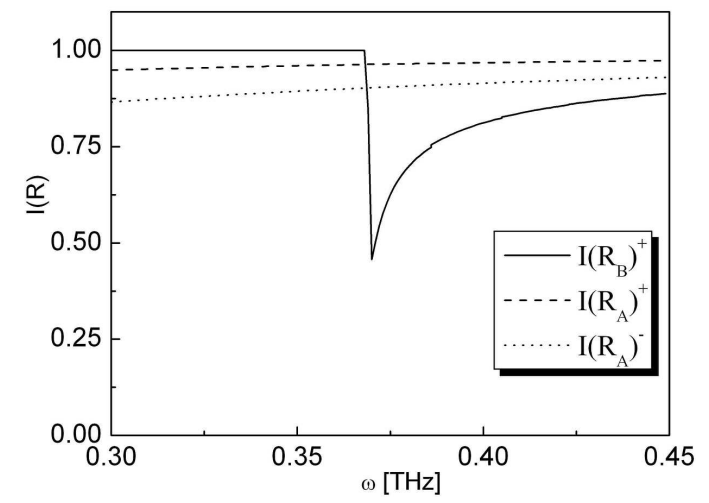

Fig. 3. Dependence of reflection intensity, for each component of reflected wave, on the magnetoelastic wave frequency at $A=5 \times 10^{-7} \mathrm{~cm}$. All other parameters are the same as in Fig. 1.

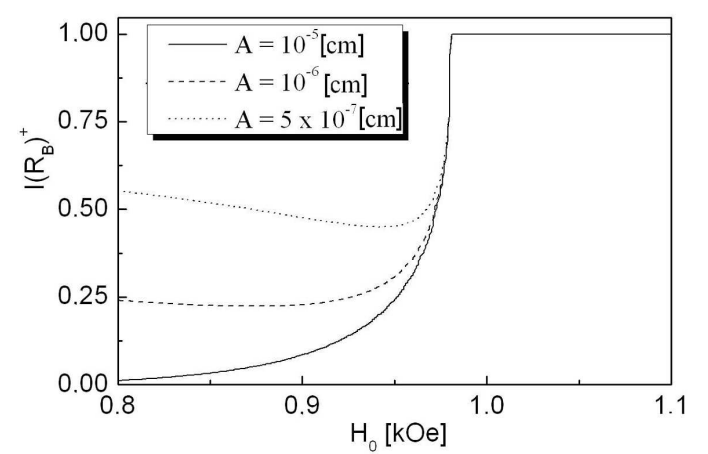

Fig. 4. Dependence of intensity of reflected wave on the value of the external uniform permanent magnetic field at $\omega=0.369 \mathrm{THz}$. All other parameters are the same as in Fig. 3.

$A \sim \alpha / d$, where $d$ is the interface effective thickness. The smaller is the value of the parameter $A$, the weaker is the coupling in the interface and, accordingly, the greater is the reflection amplitude.

To determine the reflection and transmission amplitudes (for magnetic subsystem), we will use boundary conditions that can be obtained by integrating the equation of the magnetic moment dynamics on the boundary $z=0$ taking into consideration the expression (9):

$$
\begin{aligned}
& {\left[A\left(\mu_{2}^{ \pm}-\gamma \mu_{1}^{ \pm}\right)+\alpha_{1}\left(\mu_{1}^{ \pm}\right)^{\prime}\right]_{z=0}=0} \\
& {\left[A\left(\gamma \mu_{1}^{ \pm}-\mu_{2}^{ \pm}\right)-\alpha_{2} \gamma\left(\mu_{2}^{ \pm}\right)^{\prime}\right]_{z=0}=0}
\end{aligned}
$$

where $\gamma=M_{02} / M_{01}$.

Thus, the reflection and transmission amplitudes can be written as follows:

$$
\begin{aligned}
\left(R^{ \pm}\right)_{A, B} & =\frac{\gamma \alpha_{1} \alpha_{2}\left(k_{2}^{ \pm}\right)_{A, B}-\mathrm{i} A\left(\alpha_{1}-\gamma^{2} \alpha_{2}\left(n_{2}^{ \pm}\right)_{A, B}\right)}{\gamma \alpha_{1} \alpha_{2}\left(k_{2}^{ \pm}\right)_{A, B}-\mathrm{i} A\left(\alpha_{1}+\gamma^{2} \alpha_{2}\left(n_{2}^{ \pm}\right)_{A, B}\right)} \\
\left(D^{ \pm}\right)_{A, B} & =\frac{-2 A \mathrm{i} \alpha_{1}}{\gamma \alpha_{1} \alpha_{2}\left(k_{2}^{ \pm}\right)_{A, B}-\mathrm{i} A\left(\alpha_{1}+\gamma^{2} \alpha_{2}\left(n_{2}^{ \pm}\right)_{A, B}\right)}
\end{aligned}
$$

The intensities of reflected and transmitted waves are determined as the relations of flux densities of reflected or transmitted wave to the flux density of an incident wave [15]. So, they are

$$
\begin{aligned}
& I\left(R_{A, B}\right)^{ \pm}=\left|\left(R^{ \pm}\right)_{A, B}\right|^{2}, \\
& I\left(D_{A, B}\right)^{ \pm}=\frac{\alpha_{2}}{\alpha_{1}} \operatorname{Re}\left(n_{2}^{ \pm}\right)_{A, B}\left|\left(D^{ \pm}\right)_{A, B}\right|^{2} .
\end{aligned}
$$

Figures 1-3 represent the dependences of intensities of different components of reflected wave $I(R)$ on the frequency $\omega$ with values of material parameters that are ordinary for garnet ferrites, such as $\mathrm{Y}_{3} \mathrm{Fe}_{5} \mathrm{O}_{12}$ and $\mathrm{Sm}_{3} \mathrm{Fe}_{5} \mathrm{O}_{12}$ [16], at three different values of $A$. The component, for which $\left(k_{1}^{-}\right)_{B}^{2}<0$, does not propagate due to quick damping in the first medium. There are such val- 
ues of frequencies, at which intensities of different components greatly differ from each other. This fact allows to control the intensities of waves of different branches. It is important to note that the range of frequencies in Fig. 1, at which the value of reflection intensity changes from 1 to about 0 , is narrow and it is possible to substantially change the reflectivity of this structure in case of changing the frequency value to the amount of $\approx 1 \mathrm{GHz}$, that can be used to create sensors of magnetoelastic waves.

The value of the magnetic field can substantially change the reflection nature of magnetoelastic waves, which is shown in Fig. 4. The specific dependence of intensity of a reflected wave on the value of the external magnetic field makes possible to control the reflection process of magnetoelastic waves in a wide range by changing only the value of the external magnetic field, at constant parameters of the material.

It is necessary to note that as shown in Figs. 1-4, the reflection intensity substantially depends on the value of the parameter $A$ characterizing the coupling in the interface, and it is especially important at small values of this parameter.

\section{Summary}

We have found that the intensity of reflection of magnetoelastic waves from the boundary of two uniform ferromagnets is different for each branch of magnetoelastic waves. The position of the resonance region (near the point of total transmission for either branch) depends sensitively upon the wave frequency and the value of external magnetic field. Besides, the intensity of reflection depends essentially on the parameter characterizing the coupling in the interface between uniform ferromagnetic parts.

\section{References}

[1] Yu.V. Gulyaev, P.E. Zil'berman, Russ. Phys. J. 31, 860 (1988).

[2] J.P. Parekh, H.L. Bertoni, J. Appl. Phys. 45, 434 (1974).

[3] P.R. Emtage, Phys. Rev. 13, 3063 (1976).

[4] B. Laks, D.L. Mills, Phys. Rev. B 22, 4445 (1980).

[5] Z.V. Gareeva, R.A. Doroshenko, S.V. Seregin, Phys. Metals Phys. Metallurgy 103, 488 (2007).

[6] M.N. Seavey, Phys. Rev. 170, 560 (1968).

[7] O.S. Tarasenko, S.V. Tarasenko, V.M. Yurchenko, Fiz. Tverd. Tela 46, 2200 (2004) [Phys. Sol. Stat. 46, 2275 (2004)].

[8] M.M. Karpuk, D.A. Kostuk, Yu.A. Kuzavko, V.G. Shavrov, Zh. Tekh. Fiz. 73, 97 (2003).

[9] M.M. Karpuk, D.A. Kostyuk, Yu.A. Kuzavko, V.G. Shavrov, Pis'ma Zh. Tekhn. 29, 86 (2003) [Tech. Phys. Letters. 29, 743 (2003)].

[10] Yu.A. Kuzavko, V.G. Shavrov, Akust. Zh. 39, 1088 (1993) [Acoust. Phys. 39, 572 (1993)].

[11] Y. Takemura, S. Masuda, T. Yamada, K. Kakuno, J. Appl. Phys. 79, 4653 (1996).

[12] A.I. Akhiezer, V.G. Baryakhtar, S.V. Peletminskii, Spin Waves, Nauka, Moscow 1967.

[13] P.M. Mors, H. Feshbach, Methods of Theoretical Physics, Izd. In. Lit., Moscow 1960.

[14] Yu.A. Kravtsov, Yu.I. Orlov, Geometrical Optics in Inhomogeneous Media, Nauka, Moscow 1980.

[15] L.D. Landau, Ye.M. Lifshitz, Quantum Mechanics, Nauka, Moscow 1989.

[16] A.H. Eschenfelder, Magnetic Bubble Technology, Mir, Moscow 1983. 